\title{
Lidil
}

Revue de linguistique et de didactique des langues

$58 \mid 2018$

L'enseignement et l'apprentissage de l'écrit académique à l'aide de corpus numériques

\section{Laurent Gautier (éd.), Figement et discours spécialisés}

Forum für Fachsprachen-Forschung, vol. 105, Berlin, Frank \& Timme, Verlag für wissenschaftliche Literatur, 2018, 158 p.

\section{Carole Calistri}

\section{OpenEdition}

\section{Journals}

Édition électronique

URL : http://journals.openedition.org/lidil/5235

DOI : $10.4000 /$ lidil.5235

ISSN : 1960-6052

Éditeur

UGA Éditions/Université Grenoble Alpes

Édition imprimée

ISBN : 978-2-37747-064-8

ISSN : $1146-6480$

Référence électronique

Carole Calistri, «Laurent Gautier (éd.), Figement et discours spécialisés », Lidil [En ligne], 58 | 2018, mis en ligne le 02 novembre 2018, consulté le 24 septembre 2020. URL : http://journals.openedition.org/ lidil/5235 ; DOI : https://doi.org/10.4000/lidil.5235

Ce document a été généré automatiquement le 24 septembre 2020.

(c) Lidil 


\title{
Laurent Gautier (éd.), Figement et discours spécialisés
}

\author{
Forum für Fachsprachen-Forschung, vol. 105, Berlin, Frank \& Timme, \\ Verlag für wissenschaftliche Literatur, 2018, 158 p.
}

\section{Carole Calistri}

\section{RÉFÉRENCE}

Laurent Gautier (éd.), Figement et discours spécialisés, Forum für Fachsprachen-Forschung, vol. 105, Berlin, Frank \& Timme, Verlag für wissenschaftliche Literatur, 2018, 158 p.

1 L'ouvrage coordonné par Laurent Gautier propose une exploration très complète du figement: sa description et son fonctionnement dans les langues de spécialité / spécialisées à travers 7 articles rassemblés pour parcourir d'un bout à l'autre ses réalisations, c'est-à-dire en considérant l'inscription du figement depuis le glossaire jusqu'au discours. Deux avancées importantes dans le domaine sont actées : d'une part, le dépassement de l'opposition stérile entre langue générale ou commune et langue de spécialité et, d'autre part, l'infléchissement, dans les recherches présentées, vers des approches plus quantitatives que qualitatives (L. Gautier) rendues possibles par l'accession à de grandes masses de données. On notera la diversité des corpus sollicités (dans trois langues) par les différentes rubriques: ils sont issus de la presse de vulgarisation scientifique (M. Iakushevich), des pages sportives (R. Vanoudheusden) ou non (R. Marti Solano) de quotidiens, mais également de glossaires, touchant à la gestion $\mathrm{du}$ risque des catastrophes naturelles (G. Gréciano), à l'économie et à l'énergie nucléaire (G. Petit), à la logistique et aux transports (J.-M. Delagneau) ou à celui de la faim dans le monde (F. Rigat).

2 Une deuxième dimension est constituée par l'articulation entre les unités considérées par chacun des articles et leurs usages dans un cadre qui les englobe et en légitime l'étude. À chacun des niveaux, les auteurs combinent la précision dans la description de l'objet délimité (unité polylexicale, collocation, phraséologie, stéréotypie) avec les situations 
d'emploi, dans une synergie qui redéfinit la distinction entre langue et discours. On va de la limite phrastique - terminologies, glossaires, collocation (M. Iakushevich), distinguée à l'intérieur de l'ensemble des phraséologies par son degré très faible d' idiomatisme - jusqu'à son au-delà, avec le texte / le discours journalistique général (R. Marti Solano) ou spécialisé (thématique de la dépression pour M. Iakushevich, du sport pour R. Vanouheusden). Choisir comme objet de réflexion le figement implique nécessairement de s'intéresser à ce qui n'est pas figé / n'est pas considéré comme figé ou non entièrement figé, ou non toujours figé. Les auteurs envisagent également les mouvements du figement, par exemple celui qui reflue de la polylexicalité à une certaine monolexicalité ou bien qui en dilue le projet, faute d'une convergence des instances de légitimation (G. Petit), ou celui qui prend en compte l'usure de l'usage, le figement appelant le défigement en synchronie ou en diachronie à travers différentes manipulations phraséologiques (R. Marti Solano).

Une troisième caractéristique de l'ouvrage est le fait que c'est vers l'action qu'est dirigé le glossaire multilingue de la gestion du risque : celles de l'information, de la formation, de l'enseignement en vue de la protection des hommes et de leurs biens (G. Gréciano), de la même manière que les collocations relatives à la dépression qui construisent la cohérence de la présentation des symptômes fournissent de quoi identifier la maladie, dans une perspective prophylactique (M. Iakushevich). Il est également montré que l'étude du phénomène du figement est ou peut être une arme pédagogique et économique pour contrebalancer la concurrence ou l'imposition d'une lingua franca - l'anglais. En effet, la neutralité de la lingua franca obère la réalité du savoir spécialisé des langues-cultures concernées par les échanges (J.-M. Delagneau). La dimension politique, dans son sens le plus noble, celui qui forme la réflexion du citoyen et veille à l'intérêt commun, est montrée / démontrée dans la fine étude de la langue de bois des institutions internationales relativement aux différentes désignations de la faim, devenue «insécurité alimentaire» (F. Rigat); dimension politique également avec l'analyse du babélisme montrant l'absence de concertation d'institutions publiques (G. Petit). La poésie ne manque pas à l'appel avec l'étude de la variation stylistique et de la créativité phraséologique à l'œuvre dans le domaine journalistique (R. Marti-Solano).

On appréciera dans ce volume le grain des analyses, corollaire de la complexité explorée, qui en fera un glossaire sûr et précis pour les étudiants. Cet ouvrage fait la preuve de la vitalité de la recherche terminologique / terminographique, et convainc en outre de son impact philosophique, social, politique.

\section{AUTEURS}

\section{CAROLE CALISTRI}

LINE, Université - ESPE de Nice 